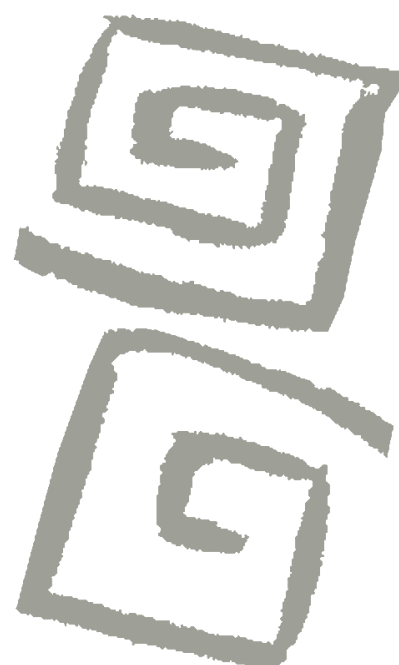

\title{
Análisis del impacto de la mortalidad por suicidios en México, 2000-2012
}

\author{
Analysis of the impact of mortality due to \\ suicides in Mexico, 2000-2012
}

Dávila Cervantes, Claudio Alberto ; Ochoa Torres, María del Pilar²; Casique Rodríguez, Irene ${ }^{3}$

${ }^{1}$ Doctor en Estudios de Población. ProfesorInvestigador, Facultad Latinoamericana de Ciencias Sociales (FLACSO), México. claudio.davila@flacso.edu.mx

${ }^{2}$ Maestra en Población y Desarrollo. Asistente de investigación, Centro de Investigaciones y Estudios Superiores en Antropología Social (CIESAS), México. pilar.ochoa@flacso.edu.mx

${ }^{3}$ Doctora en Sociología. Investigadora titular, Centro Regional de Investigaciones Multidisciplinarias,

Universidad Nacional Autónoma de México (CRIMUNAM), México.

irene@correo.crim.unam.mx
RESUMEN El objetivo del presente artículo es analizar la carga de la mortalidad por suicidios en México, a partir del indicador de años de vida perdidos (AVP), entre 2000 y 2012, por sexo, grupos de edad (para menores de 85 años de edad) a nivel nacional y por entidad federativa. Se emplearon estadísticas vitales de mortalidad y estimaciones de población para calcular tasas estandarizadas de mortalidad y los años de vida perdidos por suicidios. Entre 2000 y 2012 se dio un incremento sostenido de la tasa de mortalidad por suicidios. En hombres, el grupo de edad con las mayores tasas fue el de 85 años y más; para mujeres el de 15-19 años. El mayor impacto en la esperanza de vida se dio entre los 20 y 24 años en hombres y entre los 15 y 19 años para mujeres. Los estados con la mayor mortalidad se ubican en la península de Yucatán (Yucatán, Quintana Roo y Campeche). La mortalidad por suicidio en México se ha incrementado de manera constante. Los suicidios son prevenibles, por lo que es fundamental implementar políticas públicas en salud a través de acciones de identificación oportuna, estrategias integrales de prevención y el estudio detallado de los factores de riesgo asociados.

PALABRAS CLAVES Mortalidad; Suicidio; Tasa de Mortalidad; Años de Vida Perdidos; México.

ABSTRACT The objective of this study was to analyze the burden of disease due to suicide in Mexico using years of life lost (YLL) between 2000 and 2012 by sex, age group (for those under 85 years of age) and jurisdiction. Vital statistics on mortality and population estimates were used to calculate standardized mortality rates and years of life lost due to suicide. Between 2000 and 2012 a sustained increase in the suicide mortality rate was observed in Mexico. The age group with the highest rate was 85 years of age or older for men, and 15-19 years of age for women. The highest impact in life expectancy due to suicide occurred at 20 to 24 years of age in men and 15 to 19 years of age in women. The states with the highest mortality due to suicide were located in the Yucatan Peninsula (Yucatan, Quintana Roo and Campeche). Mortality due to suicide in Mexico has increased continually. As suicides are preventable, the implementation of health public policies through timely identification, integral prevention strategies and the detailed study of associated risk factors is imperative.

KEY WORDS Mortality; Suicide; Mortality Rate; Years of Life Lost; Mexico. 


\section{INTRODUCCIÓN}

El suicidio se define como el acto autoinfligido para causarse la muerte en forma voluntaria, deliberada, en el que intervienen sucesivamente tres etapas, Ilamadas en conjunto proceso suicida: el deseo suicida, la idea suicida y el acto suicida en sí (1). Este acto es tan antiguo como la misma humanidad y sus características son dinámicas, varían en gran medida de acuerdo a la cultura (2) y a las circunstancias contextuales, temporales, geográficas y personales (3). A nivel poblacional, el suicidio se ha estudiado a partir de enfoques como el antropológico, psicológico, sociológico y epidemiológico (4).

La Organización Mundial de la Salud (OMS) (5) establece que el suicidio es considerado mundialmente un problema de salud pública muy importante que, a pesar de ser en gran medida prevenible, provoca casi la mitad de todas las muertes violentas para hombres y más del $70 \%$ para mujeres, lo que se traduce en casi un millón de víctimas al año, con costos económicos cifrados en miles de millones de dólares. En el año 2000 se reportaron aproximadamente 815.000 suicidios a nivel mundial, lo cual representó una tasa anual de 14,5 suicidios por cada 100.000 habitantes (6); mientras que, en 2012, se produjo un leve descenso, con una tasa anual mundial de suicidio de 11,4 por 100.000 habitantes aproximadamente $(15,0$ entre hombres y 8,0 entre mujeres) (5). En 2012 el suicidio representaba el 1,4\% del total de defunciones en todo el mundo y se ubicaba como la decimoquinta causa principal de muerte. Se ha estimado que cada 40 segundos una persona se suicida en alguna parte del mundo y que de estas muertes un gran porcentaje pertenece a población joven, tanto así que a nivel mundial el suicidio es la segunda causa principal de muerte en el grupo de 15 a 29 años de edad (5). Entre los adultos de 30 a 49 años de edad representa el 4,1\% del total de muertes y se clasifica como la quinta causa principal de muerte a nivel mundial. Ante todo ello, es importante reconocer que los suicidios son un grave problema de salud pública, y no únicamente en los países desarrollados, como se consideraba en el pasado, sino que la mayoría de los suicidios se cometen en países de ingresos bajos y medianos, en los que los recursos y servicios para la identificación temprana son escasos y limitados, y el tratamiento y apoyo a las personas necesitadas son insuficientes (5).

Otra característica importante a considerar de la mortalidad por esta causa es que si bien las tasas de suicidio en general son mayores para hombres, no se da de manera homogénea en todos los países. Esto se ve reflejado en que en 2012, en los países de ingresos altos, la razón hombre/mujer de las tasas estandarizadas de suicidio fue de 3,5 mientras que, en los países de ingresos bajos y medianos, la razón hombre/mujer fue considerablemente menor ( 1,6 suicidios masculinos por cada femenino, aproximadamente) (5).

En comparación con otras regiones del mundo, América Latina muestra tasas bajas de suicidio con un nivel de 6,1 defunciones por cada 100.000 habitantes (5). Sin embargo, en los últimos años se ha presentado un constante incremento de la mortalidad por esta causa, destacando Chile, Venezuela, Uruguay y México (7).

A pesar de que las tasas de mortalidad en México son bajas, tanto a nivel mundial como en la región (6), desde la década de 1950 se ha dado un incremento sostenido e ininterrumpido de los casos de suicidio, especialmente en la población joven, por lo que en México el suicidio se ha convertido en un problema de salud pública de gran relevancia e impacto (8-10). En 1950, las tasas de mortalidad por suicidios en México se estimaron en cerca de 1,5 suicidios por cada 100.000 habitantes; para 2008 dichas tasas se triplicaron alcanzado un nivel de poco más de 4,8 suicidios (10). Este incremento de la mortalidad por suicidios se ha observado en todas las entidades federativas, lo que da cuenta de lo extendido de este problema de salud, así como de la necesidad de analizar dicha tendencia (9), tanto a nivel nacional como estatal. Otra característica importante de este fenómeno en México se relaciona con el hecho de que la mayoría de las personas que se suicidan son hombres y estas diferencias por género han ido aumentando con el paso de los años (10), lo que resalta la importancia de investigar el fenómeno integrando esta perspectiva de mortalidad diferencial por suicidios entre hombres y mujeres.

Dentro de este marco, el objetivo principal del presente artículo es analizar la carga de la mortalidad por suicidios en México, a partir del 
indicador de años de vida perdidos (AVP), entre 2000 y 2012, por sexo, grupos de edad (para menores de 85 años de edad), a nivel nacional y por entidad federativa.

\section{MATERIAL Y MÉTODOS}

Se realizó un estudio de tipo transversal y descriptivo. Los datos de defunciones se obtuvieron del Instituto Nacional de Estadística y Geografía (INEGI), puestos a disposición por la Secretaría de Salud (SSA), para el período 2000-2012. Las estimaciones de población a nivel nacional y por entidad federativa, así como el índice de marginación estatal (a) se tomaron del Consejo Nacional de Población (CONAPO). Se consideraron los faIlecimientos por suicidios según los criterios de la Clasificación Internacional de Enfermedades (CIE-10), que aparecen en el rubro "Otras causas externas de mortalidad" (códigos X60-X84), y se realizó un prorrateo para distribuir las muertes de las edades no especificadas entre el resto de los grupos de edad.

Para el análisis, únicamente se tuvieron en cuenta las defunciones por suicidios ocurridas en el interior del país, quedando excluidos los siguientes casos: 1) muertes ocurridas en el exterior (6 registros); 2) las defunciones de mayores de 85 años (52 casos) para el cálculo de los AVP. A partir de esto, se descartó el 0,12\% del total de registros para el cálculo de las tasas estandarizadas de mortalidad y un 1,03\% para el cálculo de los AVP.

Se calcularon las tasas estandarizadas de mortalidad por suicidios, por sexo y grupos de edad a nivel nacional para el periodo comprendido entre 2000 y 2012, y a nivel estatal para 2010, utilizando la población nacional de 2010 como estándar. De igual forma, se calcularon los AVP propuestos por Arriaga (11), para estimar cuántos años de vida en promedio se perdieron entre 2000 y 2012 por suicidios de personas menores a 85 años de edad o, dicho en otras palabras, para determinar cuántos años más deberían haber vivido las personas que fallecieron por esta causa antes de los 85 años (11). Se utilizó el supuesto de mortalidad nula entre las dos edades elegidas, es decir, que aquellos que mueren deberían haber vivido hasta los 85 años de edad (b). Este supuesto del método tiene la ventaja de que, al comparar la mortalidad observada con la mortalidad hipotética nula, las comparaciones quedan estandarizadas y, por lo tanto, se pueden realizar comparaciones históricas o entre poblaciones (11). El otro supuesto importante del método de Arriaga se basa en asumir que la distribución de las defunciones por causa de muerte de la tabla de mortalidad es igual a la observada en las defunciones registradas para cada grupo de edad (11) (c). Se seleccionó este rango etario debido a que para este indicador se recomienda tomar, en todos los casos, la edad más alta posible siempre que las estadísticas de defunciones por causas sean fehacientes (11). Esta técnica es considerada como una de las principales herramientas para medir los cambios en el nivel de la mortalidad y la mortalidad por causas; es además un indicador útil, ya que permite relacionar la mortalidad por suicidios con el cambio de la esperanza de vida temporaria entre edades seleccionadas (10) dando cuenta de los cambios y del impacto de la mortalidad por esta causa en la salud de la población (12). Finalmente, se realizó un análisis de correlación entre las tasas de mortalidad por suicidios y el índice de marginación estatal, con la intención de analizar si existe alguna relación entre el nivel socioeconómico y la mortalidad por suicidios, ya que previamente otros estudios han relacionado la mortalidad por esta causa con la situación socioeconómica de los países (5).

\section{Limitaciones}

La información sobre suicidios se basó en las estadísticas vitales de mortalidad. En este sentido, el registro de suicidios es un procedimiento complejo, de niveles múltiples, que abarca cuestiones médicas y legales e incluye a varias autoridades responsables (5). En el caso mexicano, los registros se generan a partir de los certificados de defunción, en los que se asientan, para cada caso, datos relativos a la presunción respecto a si la causa de la defunción fue por accidente, agresión o suicidio, según la opinión inicial del médico legista o forense, lo cual queda asentado en la averiguación previa correspondiente por parte del Ministerio Público (8). La conclusión de dicha averiguación, 
o bien del proceso penal, ratifica o rectifica la presunción considerada en un principio, resultado que ya no se refleja en las estadísticas elaboradas con los certificados de defunción (8), por lo que muy probablemente exista un subregistro de la información aquí utilizada. Esta limitación de la fuente de datos sobre la mortalidad no es exclusiva del suicidio, pero dada la sensibilidad de este, es probable que haya más problemas de subregistro y mala clasificación que en otras causas. Aunado a ello, las estadísticas vitales de mortalidad tienen otro tipo de limitaciones, como la falta de uniformidad en las definiciones del registro, una cobertura inadecuada en regiones poco accesibles, errores de omisión, subregistro, y retraso en la publicación de la información.

Una limitante adicional es que la estimación de los AVP se ve restringida al tener que delimitarse un rango de edad, en este caso en particular entre 10 y 85 años, lo que provoca que se subestime el impacto total de la mortalidad por suicidios. Esta desventaja se reduce considerablemente a medida que aumenta la edad del grupo abierto. Es por ello que se tomó un rango de edad tan amplio en este estudio, lo que implicó que la mayor parte de los individuos, cuya muerte se debió a esta causa, quedó incluida en esta investigación.

\section{RESULTADOS}

En 2000 ocurrieron un total de 3.475 suicidios consumados en México, de los cuales el $84,5 \%$ fueron llevados a cabo por hombres y el $15,5 \%$ por mujeres. Para 2012 esta cifra se incrementó a 5.550 suicidios consumados $(80,5 \%$ masculinos y $19,5 \%$ femeninos). En cuanto a la relación hombre/mujer de las defunciones por esta causa se observa que, en 2000, esta proporción era cercana a 5,5 suicidios masculinos por cada femenino, cifra que fue disminuyendo hasta encontrarse en un valor poco menor a 4,2 en 2012 (Figura 1). En cuanto a la tendencia de la mortalidad por suicidios, se puede observar que entre 2000 y 2012 se presentó un incremento sostenido, reflejado en el aumento paulatino de las tasas de mortalidad, las cuales pasaron de 6,2 suicidios para hombres y de 1,1 para mujeres por 100.000 habitantes, en 2000, a un nivel de 7,8 suicidios masculinos y 1,8 suicidios femeninos (Figura 1). Estos cambios representan un aumento del $25,8 \%$ en hombres y del $68,4 \%$ en mujeres en el periodo analizado.

Las edades en las que se concentra la mayoría de los suicidios son entre los 15 y los 49 años,

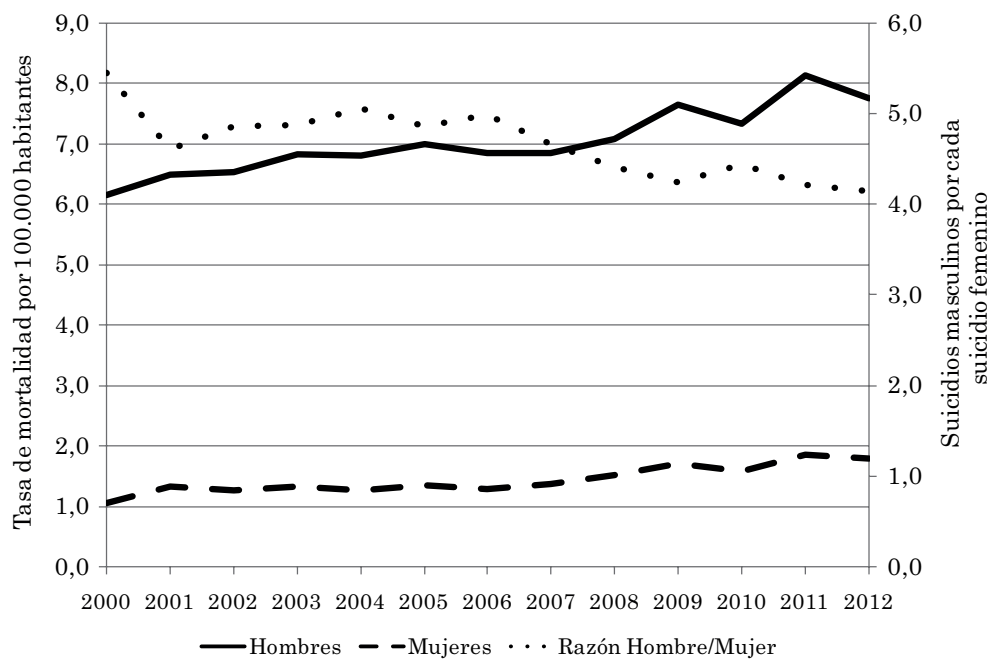

Figura 1. Tendencia de las tasas de mortalidad por suicidios ocurridos según edad y sexo. México, 2000-2012.

Fuente: Elaboración propia a partir de estadísticas vitales de mortalidad del Instituto Nacional de Estadística y Geografía (INEGI) y de proyecciones de población 2010-2030 del Consejo Nacional de Población (CONAPO). 


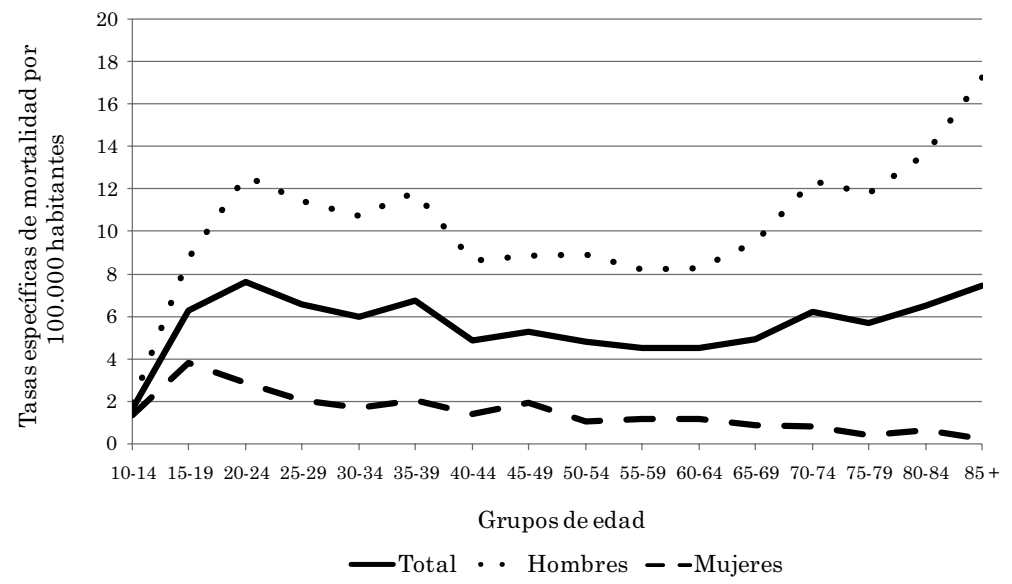

Figura 2. Tasa estandarizada de mortalidad por suicidios ocurridos según edad y sexo. México, 2010.

Fuente: Elaboración propia a partir de estadísticas vitales de mortalidad del Instituto Nacional de Estadística y Geografía (INEGI) y de proyecciones de población 2010-2030 del Consejo Nacional de Población (CONAPO).

encontrándose en este grupo etario el $77 \%$ del total de suicidios masculinos y el $79 \%$ del total de suicidios femeninos ocurridos entre 2000 y 2012. Respecto a la proporción que los suicidios ocupan dentro de las muertes violentas para hombres, los suicidios han representado, desde 2000, entre un $7,4 \%$ y un $9,1 \%$ del total de defunciones por estas causas; mientras que en el caso de las mujeres ha prevalecido una tendencia creciente, pasando del $5,5 \%$ del total de defunciones por causas violentas en 2000 a poco más del 8,5\% en 2012.

Una característica de la mortalidad por suicidios entre sexos se refiere al impacto diferencial por edad. Para ejemplificar este comportamiento se analizan las tasas estandarizadas de suicidios por grupos de edad, para hombres y mujeres a nivel nacional para el año 2010 (Figura 2). En el caso de hombres la tasa estandarizada de suicidios por edad tiene un comportamiento singular, ya que alcanza un valor alto en el grupo de edad de 20 a 24 años, para después descender de manera paulatina hasta los 65 años, edad a partir de la cual la tasa se incrementa hasta alcanzar su máximo valor en el grupo de edad de 85 años y más. Para mujeres la situación es distinta puesto que, una vez que las tasas de mortalidad alcanzan su máximo en el grupo de 15 a 19 años, presentan una tendencia a disminuir de manera continua en todo el rango de edad (Figura 2).
Para las entidades federativas del país, en 2010, aquellas con las mayores tasas estandarizadas de mortalidad masculina fueron Yucatán, Tabasco, Quintana Roo y Campeche (con tasas de 16,$8 ; 15,6 ; 15,5$ y 13,1 suicidios por cada 100.000 hombres respectivamente) (Figura 3 y Figura 4); mientras que, para mujeres, las principales entidades fueron Yucatán, Quintana Roo y San Luis Potosí (alcanzando tasas de 3,5; 3,0 y 2,6 suicidios por cada 100.000 mujeres respectivamente).

Esto es interesante ya que, tanto para hombres como para mujeres, la región del país con las tasas de suicidios más altas es la península de Yucatán. Por otro lado, aquellos estados con las menores tasas de mortalidad por esta causa fueron Chiapas, Guerrero, Morelos, Hidalgo y Tlaxcala para hombres (con cifras de 3,5; 3,7; 4,0; 4 ,0 y 4,3 suicidios respectivamente); y en el caso de mujeres fueron Chiapas, Baja California Sur, Zacatecas, Hidalgo y Baja California (con tasas de suicidios de 0,$4 ; 0,6 ; 0,7 ; 0,7$ y 0,9 respectivamente) (Figura 5). Los resultados del análisis de correlación entre las tasas de mortalidad por suicidios y el índice de marginación estatal muestran un coeficiente de correlación negativo $(-0,25)$ y que no resultó estadísticamente significativo $(p>0,16)$, indicando que no existe una asociación lineal entre el nivel socioeconómico de las entidades federativas y la tasa de mortalidad por suicidios a nivel estatal. 


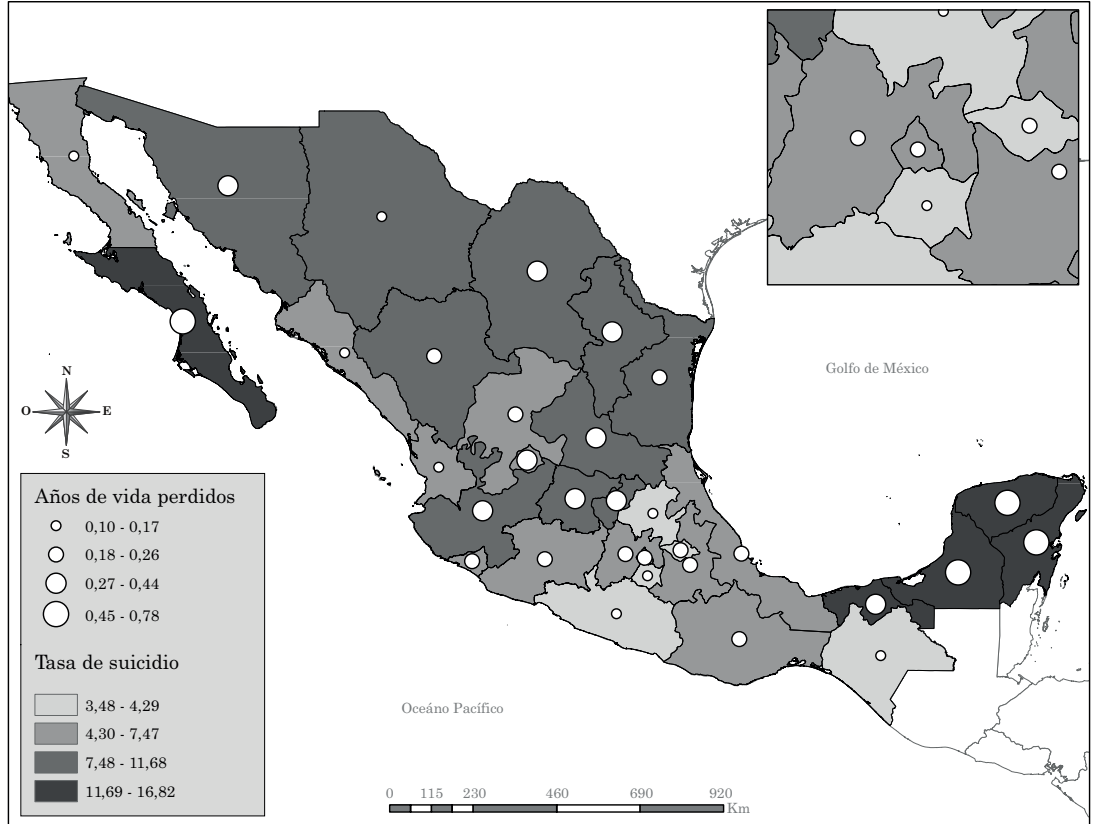

Figura 3. Tasas de mortalidad y años de vida perdidos por suicidio en hombres. México, 2010.

Fuente: Elaboración propia a partir de estadísticas vitales de mortalidad del Instituto Nacional de Estadística y Geografía (INEGI) y de proyecciones de población 2010-2030 del Consejo Nacional de Población (CONAPO).

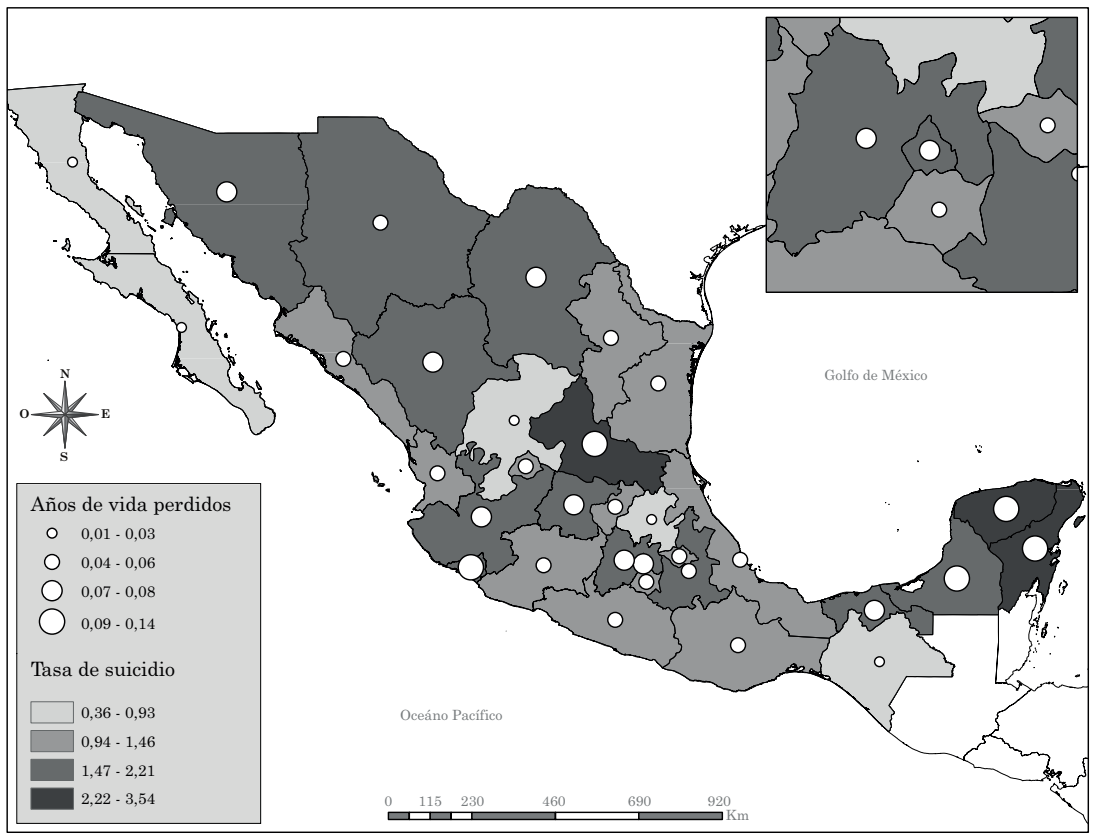

Figura 4. Tasas de mortalidad y años de vida perdidos por suicidio en mujeres. México, 2010.

Fuente: Elaboración propia a partir de estadísticas vitales de mortalidad del Instituto Nacional de Estadística y Geografía (INEGI) y de proyecciones de población 2010-2030 del Consejo Nacional de Población (CONAPO). 
Cuadro 1. Años de vida perdidos por suicidios ocurridos en hombres, según edad. México, 2000-2012.

\begin{tabular}{|c|c|c|c|c|c|c|c|c|c|c|c|c|c|}
\hline Edad & 2000 & 2001 & 2002 & 2003 & 2004 & 2005 & 2006 & 2007 & 2008 & 2009 & 2010 & 2011 & 2012 \\
\hline $0-4$ & 0,00000 & 0,00000 & 0,00000 & 0,00000 & 0,00000 & 0,00000 & 0,00000 & 0,00000 & 0,00000 & 0,00000 & 0,00000 & 0,00000 & 0,00000 \\
\hline 5-9 & 0,00000 & 0,00000 & 0,00000 & 0,00000 & 0,00000 & 0,00000 & 0,00000 & 0,00000 & 0,00000 & 0,00000 & 0,00000 & 0,00000 & 0,00000 \\
\hline $10-14$ & 0,00398 & 0,00449 & 0,00569 & 0,00536 & 0,00618 & 0,00612 & 0,00691 & 0,00529 & 0,00719 & 0,00656 & 0,00701 & 0,00932 & 0,00817 \\
\hline $15-19$ & 0,02845 & 0,03104 & 0,02889 & 0,03170 & 0,03270 & 0,03085 & 0,03005 & 0,02918 & 0,02960 & 0,02934 & 0,02827 & 0,03498 & 0,03522 \\
\hline $20-24$ & 0,04402 & 0,04638 & 0,04824 & 0,05143 & 0,05061 & 0,04933 & 0,04842 & 0,04642 & 0,04344 & 0,03889 & 0,03744 & 0,04336 & 0,04101 \\
\hline $25-29$ & 0,03793 & 0,03793 & 0,03787 & 0,04083 & 0,04234 & 0,04439 & 0,04209 & 0,04073 & 0,03780 & 0,03485 & 0,03084 & 0,03508 & 0,03613 \\
\hline 30-34 & 0,02669 & 0,03210 & 0,03070 & 0,03319 & 0,03257 & 0,03425 & 0,03350 & 0,03513 & 0,03101 & 0,02958 & 0,02599 & 0,02817 & 0,02963 \\
\hline 35-39 & 0,02148 & 0,01914 & 0,02180 & 0,02252 & 0,02641 & 0,02584 & 0,02732 & 0,02575 & 0,02571 & 0,02614 & 0,02590 & 0,02494 & 0,02623 \\
\hline 40-44 & 0,01294 & 0,01507 & 0,01656 & 0,01832 & 0,01806 & 0,01953 & 0,01592 & 0,01878 & 0,01832 & 0,01877 & 0,01665 & 0,02101 & 0,02004 \\
\hline $45-49$ & 0,01336 & 0,01413 & 0,01323 & 0,01348 & 0,01408 & 0,01473 & 0,01542 & 0,01453 & 0,01429 & 0,01760 & 0,01462 & 0,01667 & 0,01447 \\
\hline $50-54$ & 0,01063 & 0,01164 & 0,01084 & 0,01077 & 0,01102 & 0,01233 & 0,01223 & 0,01150 & 0,01142 & 0,01293 & 0,01245 & 0,01329 & 0,01182 \\
\hline 55-59 & 0,00829 & 0,00827 & 0,00853 & 0,00955 & 0,00984 & 0,00944 & 0,01042 & 0,00750 & 0,00818 & 0,01069 & 0,00911 & 0,00971 & 0,00904 \\
\hline $60-64$ & 0,00604 & 0,00758 & 0,00655 & 0,00701 & 0,00729 & 0,00603 & 0,00635 & 0,00650 & 0,00847 & 0,00787 & 0,00700 & 0,00863 & 0,00820 \\
\hline $65-69$ & 0,00478 & 0,00584 & 0,00524 & 0,00614 & 0,00637 & 0,00495 & 0,00600 & 0,00689 & 0,00685 & 0,00564 & 0,00575 & 0,00631 & 0,00558 \\
\hline $70-74$ & 0,00444 & 0,00338 & 0,00416 & 0,00348 & 0,00320 & 0,00430 & 0,00419 & 0,00473 & 0,00349 & 0,00443 & 0,00467 & 0,00460 & 0,00403 \\
\hline 75-79 & 0,00172 & 0,00322 & 0,00232 & 0,00300 & 0,00272 & 0,00238 & 0,00222 & 0,00210 & 0,00189 & 0,00261 & 0,00216 & 0,00236 & 0,00201 \\
\hline $80-84$ & 0,00093 & 0,00100 & 0,00062 & 0,00068 & 0,00055 & 0,00087 & 0,00065 & 0,00063 & 0,00063 & 0,00052 & 0,00061 & 0,00059 & 0,00057 \\
\hline Total & 0,22567 & 0,24113 & 0,24431 & 0,25746 & 0,26394 & 0,26497 & 0,26168 & 0,25564 & 0,24828 & 0,24641 & 0,22845 & 0,25902 & 0,25214 \\
\hline
\end{tabular}

Fuente: Elaboración propia a partir de estadísticas vitales de mortalidad del Instituto Nacional de Estadística y Geografía (INEGI) y de proyecciones de población 2010-2030 del Consejo Nacional de Población (CONAPO).

Cuadro 2. Años de vida perdidos por suicidios ocurridos en mujeres, según edad. México, 2000-2012.

\begin{tabular}{|c|c|c|c|c|c|c|c|c|c|c|c|c|c|}
\hline Edad & 2000 & 2001 & 2002 & 2003 & 2004 & 2005 & 2006 & 2007 & 2008 & 2009 & 2010 & 2011 & 2012 \\
\hline $0-4$ & 0,00000 & 0,00000 & 0,00000 & 0,00000 & 0,00000 & 0,00000 & 0,00000 & 0,00000 & 0,00000 & 0,00000 & 0,00000 & 0,00000 & 0,00000 \\
\hline $5-9$ & 0,00000 & 0,00000 & 0,00000 & 0,00000 & 0,00000 & 0,00000 & 0,00000 & 0,00000 & 0,00000 & 0,00000 & 0,00000 & 0,00000 & 0,00000 \\
\hline $10-14$ & 0,00277 & 0,00395 & 0,00404 & 0,00327 & 0,00347 & 0,00376 & 0,00399 & 0,00358 & 0,00475 & 0,00516 & 0,00497 & 0,00729 & 0,00564 \\
\hline $15-19$ & 0,00950 & 0,01157 & 0,01155 & 0,01259 & 0,01064 & 0,01168 & 0,01278 & 0,00977 & 0,01169 & 0,01467 & 0,01285 & 0,01608 & 0,01476 \\
\hline $20-24$ & 0,00726 & 0,00739 & 0,00696 & 0,00782 & 0,00653 & 0,00766 & 0,00756 & 0,00928 & 0,01025 & 0,00689 & 0,00890 & 0,01150 & 0,01095 \\
\hline $25-29$ & 0,00391 & 0,00472 & 0,00426 & 0,00449 & 0,00566 & 0,00606 & 0,00471 & 0,00614 & 0,00615 & 0,00680 & 0,00607 & 0,00842 & 0,00622 \\
\hline $30-34$ & 0,00263 & 0,00415 & 0,00343 & 0,00342 & 0,00465 & 0,00398 & 0,00406 & 0,00482 & 0,00444 & 0,00500 & 0,00452 & 0,00512 & 0,00633 \\
\hline 35-39 & 0,00223 & 0,00308 & 0,00261 & 0,00355 & 0,00307 & 0,00352 & 0,00344 & 0,00352 & 0,00422 & 0,00375 & 0,00477 & 0,00423 & 0,00442 \\
\hline $40-44$ & 0,00175 & 0,00223 & 0,00285 & 0,00281 & 0,00203 & 0,00294 & 0,00229 & 0,00341 & 0,00354 & 0,00326 & 0,00290 & 0,00342 & 0,00383 \\
\hline $45-49$ & 0,00227 & 0,00268 & 0,00251 & 0,00229 & 0,00242 & 0,00250 & 0,00205 & 0,00304 & 0,00284 & 0,00338 & 0,00344 & 0,00275 & 0,00368 \\
\hline $50-54$ & 0,00140 & 0,00213 & 0,00238 & 0,00238 & 0,00208 & 0,00217 & 0,00222 & 0,00232 & 0,00184 & 0,00214 & 0,00164 & 0,00205 & 0,00208 \\
\hline $55-59$ & 0,00095 & 0,00147 & 0,00091 & 0,00160 & 0,00164 & 0,00162 & 0,00107 & 0,00096 & 0,00199 & 0,00204 & 0,00143 & 0,00154 & 0,00177 \\
\hline $60-64$ & 0,00076 & 0,00104 & 0,00118 & 0,00099 & 0,00138 & 0,00067 & 0,00101 & 0,000956 & 0,00103 & 0,00127 & 0,00109 & 0,00081 & 0,00129 \\
\hline $65-69$ & 0,00077 & 0,00091 & 0,00081 & 0,00090 & 0,00104 & 0,00062 & 0,00075 & 0,00054 & 0,00052 & 0,00062 & 0,00060 & 0,00120 & 0,00084 \\
\hline $70-74$ & 0,00038 & 0,00037 & 0,00035 & 0,00028 & 0,00045 & 0,00054 & 0,00049 & 0,00051 & 0,00015 & 0,00049 & 0,00037 & 0,00047 & 0,00065 \\
\hline 75-79 & 0,00034 & 0,00019 & 0,00027 & 0,00017 & 0,00039 & 0,00020 & 0,00012 & 0,00041 & 0,00025 & 0,00017 & 0,00010 & 0,00000 & 0,00020 \\
\hline $80-84$ & 0,00004 & 0,00012 & 0,00004 & 0,00004 & 0,00003 & 0,00006 & 0,00008 & 0,00000 & 0,00004 & 0,00000 & 0,00004 & 0,00005 & 0,00003 \\
\hline Total & 0,03697 & 0,04598 & 0,04415 & 0,04660 & 0,04547 & 0,04798 & 0,04662 & 0,04927 & 0,05397 & 0,05562 & 0,05369 & 0,06492 & 0,06270 \\
\hline
\end{tabular}

Fuente: Elaboración propia a partir de estadísticas vitales de mortalidad del Instituto Nacional de Estadística y Geografía (INEGI) y de proyecciones de población 2010-2030 del Consejo Nacional de Población (CONAPO). 


\section{Años de vida perdidos}

Los AVP presentaron, en 2000, un nivel de 0,226 y 0,037 años para hombres y mujeres respectivamente; esto implica que si se eliminara la mortalidad por esta causa, la esperanza de vida temporaria para los menores de 85 años se incrementaría en 0,226 años para hombres (Cuadro 1) y en 0,037 en mujeres (Cuadro 2). Esta situación se modifica para 2012, ya que se incrementaron a 0,252 y 0,063 para hombres y mujeres, lo que corresponde a un cambio relativo del $11,7 \%$ y del $69,6 \%$ respectivamente. Es interesante notar que, si bien la tendencia de las tasas y AVP femeninos son análogas, en el caso de hombres no se presenta un incremento constante de los AVP, a diferencia de lo que se observó con las tasas; los AVP crecen hasta 2005 y posteriormente comienzan a decrecer hasta alcanzar su mínimo en 2010, año a partir del cual se incrementan nuevamente. Esto se puede explicar debido a que, en todo el periodo, el incremento que se presentó en la mortalidad por suicidios se dio principalmente en las edades mayores; mientras que en las menores, en las que los AVP tienen un mayor peso, se presentó una ligera disminución de la mortalidad entre 2005 y 2010.

Por grupos de edad, en hombres se puede observar que el grupo etario con el mayor número de defunciones y de AVP fue el de 20-24 años en todos los años de estudio (con un promedio de 0,045 años), mientras que para mujeres fue el de 15-19 años (promedio de 0,012). Es importante resaltar que, durante el periodo de estudio, más del $81 \%$ del total de AVP para hombres, en el rango de edades considerado, ocurrió entre los 15-49 años; mientras que, en el caso de las mujeres este porcentaje ascendió a un $79 \%$.

A nivel estatal, se observa que en 2010 la mayor mortalidad por esta causa se encuentra en las entidades federativas que conforman la península de Yucatán, para ambos sexos; esto es, las tres entidades que la conforman (Yucatán, Quintana Roo y Campeche) se encuentran entre las cinco con los mayores AVP en el país $(0,78 ; 0,59$ y 0,53 años respectivamente para hombres y 0,$14 ; 0,12$ y 0,10 años respectivamente para mujeres) (Figura 3 y Figura 4). Para hombres, las otras entidades federativas con mayores AVP que el resto son Baja California Sur (0,54 años) y Tabasco (0,44 años); y en mujeres fueron Colima (0,10 años) y San Luis Potosí (0,10 años). Por otro lado, las entidades con menor número de AVP fueron Guerrero, Chiapas, Morelos y Baja California para hombres y Chiapas, Zacatecas, Hidalgo y Baja California para mujeres, con niveles inferiores a 0,13 años en hombres y 0,025 años en mujeres (Figura 6). Al igual que con

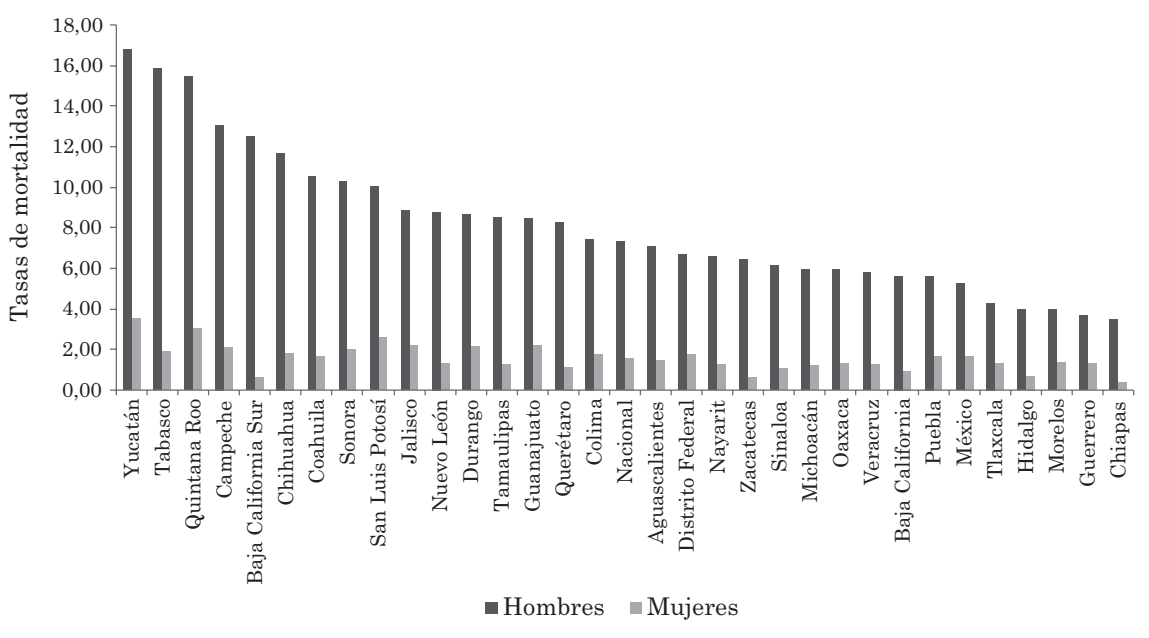

Figura 5. Tasas de mortalidad por suicidios, según sexo y entidad federativa. México, 2010.

Fuente: Elaboración propia a partir de estadísticas vitales de mortalidad del Instituto Nacional de Estadística y Geografía (INEGI) y de proyecciones de población 2010-2030 del Consejo Nacional de Población (CONAPO). 


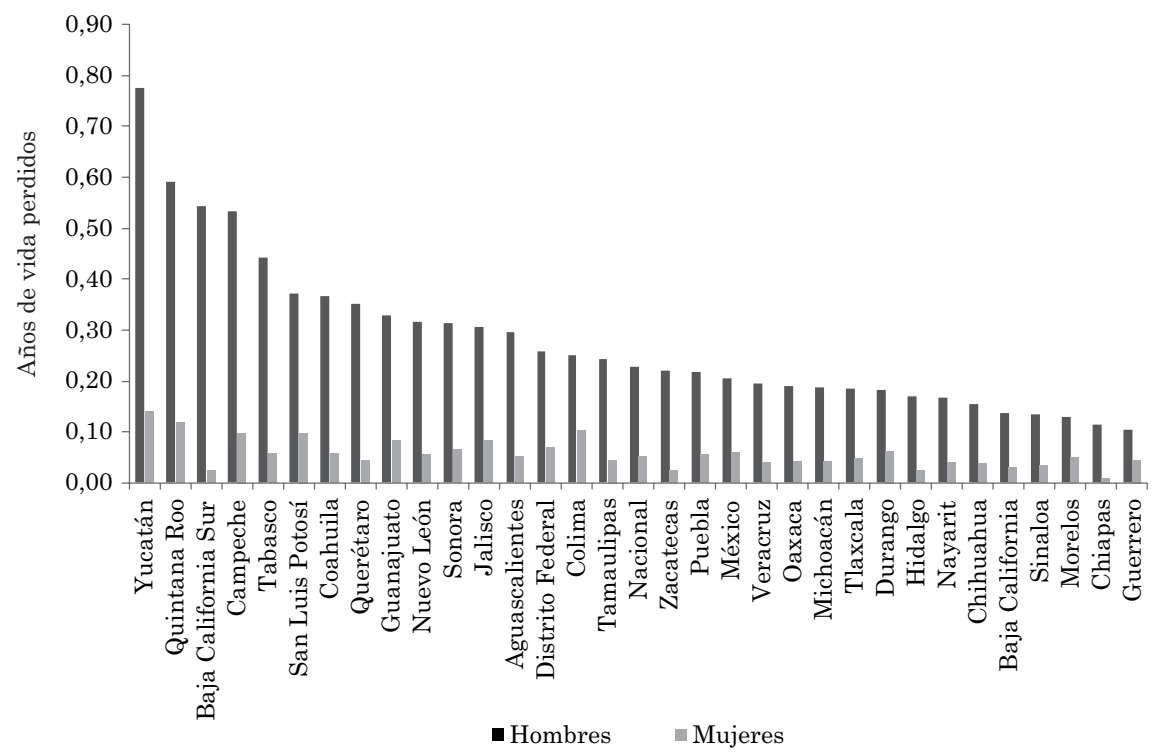

Figura 6. Años de vida perdidos por suicidios, según sexo y entidad federativa. México, 2010.

Fuente: Elaboración propia a partir de estadísticas vitales de mortalidad del Instituto Nacional de Estadística y Geografía (INEGI) y de proyecciones de población 2010-2030 del Consejo Nacional de Población (CONAPO).

las tasas de mortalidad, se calculó el coeficiente de correlación entre los AVP en las entidades federativas y el índice de marginación estatal. Los resultados de este análisis arrojaron un coeficiente de correlación negativo $(-0,19)$ que tampoco resultó estadísticamente significativo $(p>0,28)$, indicando que no existe una relación lineal entre el nivel socioeconómico de las entidades federativas y los AVP por suicidios a nivel estatal.

\section{DISCUSIÓN}

El suicidio es un importante problema de salud pública debido a su impacto en la sociedad tanto por la mortalidad prematura, que conlleva una enorme carga económica, social y psicológica para las personas, las familias y la sociedad en general (5), como por el hecho de que los intentos de suicidio dejan secuelas graves y quizá permanentes en el afectado, cuyos efectos sobre la salud de la población pueden llegar a ser de gran magnitud. Más aún, se puede argumentar que gran parte de la importancia del tema radica en que la incidencia del suicidio pudiera ser un reflejo de las malas condiciones de vida de la población (13).

La tasa de mortalidad por suicidio en México se ha incrementado de manera constante en los últimos 40 años $(10,14,15)$, lo que se corrobora en esta investigación, ya que se continúa con dicha tendencia en el periodo analizado. Sin embargo, cuando se analiza el impacto de la mortalidad por suicidios a través de los AVP, se observa una situación ligeramente distinta. Si bien hay un incremento de este indicador de 2000 a 2005, a partir de ese año la tendencia se modifica, ya que la mortalidad desciende hasta 2010, año a partir del cual se vuelve a incrementar. Esto se debe a que el aumento de la mortalidad se presentó principalmente en las edades mayores del rango de edad analizado y no de manera generalizada. Si bien el número absoluto de defunciones por suicidios fue mayor en las edades jóvenes a lo largo del periodo, las mayores tasas de suicidio se encuentran en las personas de 80 años y más; sin embargo, en algunos años se redujeron los AVP por esta causa, a diferencia de la tendencia de las tasas. Es por ello que el análisis de la mortalidad se debe realizar por medio de diferentes indicadores (10), 
como los aquí utilizados, para obtener un mejor panorama del comportamiento del fenómeno.

Por sexo se corroboró el comportamiento diferencial de la mortalidad por suicidios encontrado en otras investigaciones alrededor del tema en México $(10,14,15)$. Para hombres, las mayores tasas se localizan en el grupo de edad de 80 años y más; sin embargo, cuando se analizan los AVP por esta causa, el máximo impacto de la mortalidad por suicidios masculinos se da en el grupo de 20 a 24 años. Entre las mujeres, Hernández-Bringas y Flores-Arenales, al analizar la tendencia de los suicidios en México, encontraron que los mayores niveles de suicidio se presentan en los grupos de edad de 15 a 24 años, tanto en términos relativos como absolutos (10), cuestión que es consistente con los resultados obtenidos en esta investigación, en ambos indicadores utilizados.

Hay diversas explicaciones sobre la diferencia de las tasas de suicidio entre hombres y mujeres que se ha encontrado en esta investigación y en otras en el mismo contexto $(10,14,15)$, cuestión que se ha asociado con la "paradoja del género" (concepto que describe la evidencia de que las mujeres intentan suicidarse con más frecuencia que los hombres, pero finalmente lo concretan más los hombres que las mujeres) (16). Esto se puede basar en cuestiones de género, debido principalmente a que el significado del comportamiento suicida es distinto para uno y otro género $(6,17,18)$. Entre las principales causas, se encuentran las diferencias entre hombres y mujeres respecto de los métodos socialmente aceptables de abordar ciertas situaciones, como el estrés y el conflicto. Esto supone que los hombres se preocupan más por la desaprobación social respecto a su pensamiento y comportamiento suicida, lo que provoca que el comportamiento suicida no fatal sea poco masculino y pueda llevar a exacerbar la diferencia de género en las tasas de suicidios (19), como se observó en este estudio. Otra característica a destacar se refiere a la diferencia en los medios utilizados para suicidarse, siendo más letales en el caso de los hombres, lo cual no depende solo de la disponibilidad de acceso al método, sino de valores culturales, religiosos y sociales $(20,21)$. Esta última cuestión amerita una Ilamada de atención (15) ya que los hombres recurren a medios como el ahorcamiento y las armas de fuego, los cuales son empleados en un $78 \%$ y un $13 \%$ del total de suicidios respectivamente $(8,22)$; mientras que las mujeres prefieren utilizar otros medios como el envenenamiento por gases, vapores, alcohol o plaguicida $(8,15,22-24)$, lo que podría ser un factor que se asocia con las diferencias en la mortalidad por suicidios entre hombres y mujeres observadas en esta investigación. Asimismo, se ha sugerido que el suicidio, el consumo de alcohol y de drogas son percibidos como masculinos, mientras que el comportamiento suicida no fatal o automutilativo se considera femenino (25); lo que lleva a reflexionar que estos significados culturales pueden tener influencia en el comportamiento suicida en hombres y mujeres $(25,26)$ y, por tanto, pueden ser un factor adicional que se relacione con la diferencia de género en la mortalidad por suicidios obtenido en este estudio.

Otro factor relacionado con la diferencia de las tasas de suicidio entre hombres y mujeres es el vínculo entre el comportamiento suicida y varios desórdenes psiquiátricos, lo que se relaciona, a su vez, con diferencias de género en psicopatología (19). En este sentido, el malestar depresivo se ha identificado como uno de los factores de riesgo más importantes para la ideación suicida y se presenta de manera desigual en hombres y mujeres (27). En este contexto, debe mencionarse que la personalidad impulsiva, hostil y agresiva, relacionada con varios desordenes psiquiátricos, es más prevalente en hombres e incrementa sustancialmente el riesgo de suicidio $(28,29)$. Más aún, si bien existe una clara diferencia entre la mortalidad masculina y femenina por suicidios, los datos analizados en esta investigación y en otros estudios sobre el tema de mortalidad por suicidios en México -como el realizado por Martínez (15) y Hernández-Bringas y Flores-Arenales (10)- muestran que las tasas para las mujeres están aumentando en todo el país desde hace algunos años, lo que hace imperativo indagar, en investigaciones futuras, qué es lo que las está colocando en situaciones cada vez más riesgosas (15).

Por otro lado, se realizó el análisis a nivel estatal debido a que tanto la tasa de mortalidad como los AVP a nivel nacional son indicadores que dan una primera aproximación al tema pero, para obtener un panorama mucho más detallado sobre el fenómeno, es necesario también estudiar los datos desagregados por entidades federativas. Se corroboró que las entidades federativas con mayor 
incidencia de suicidios para ambos sexos son Yucatán, Quintana Roo y Campeche $(13,15,30)$ y se agregan, para hombres, Baja California Sur y Tabasco, y en mujeres, Colima y San Luis Potosí, como estados con un alto nivel de mortalidad por suicidios. Estos resultados por entidad federativa sugieren un área de riesgo específica formada por los estados que conforman la península de Yucatán (Figura 3 y Figura 4) (22). Por otro lado, entre aquellas entidades con bajos niveles de mortalidad por suicidios en ambos sexos, destacan Chiapas, Hidalgo y Baja California. Los resultados de esta investigación indican, también, que parece no existir una relación entre el nivel socioeconómico de las entidades federativas (aproximado a partir del índice de marginación estatal) y la mortalidad por suicidios. Esto se puede relacionar con la tendencia creciente de la mortalidad desde los años cincuenta en todas las entidades del país (10), por lo que es poco probable que los procesos de crisis económica en el país hayan tenido un efecto significativo en el incremento de la mortalidad por suicidios. Este análisis se realizó debido a que se ha argumentado que la incidencia del suicidio pudiera estar asociada con condiciones de vida deficientes de la población (13).

Los resultados obtenidos en este estudio, no solo permiten corroborar la tendencia creciente de la mortalidad por suicidios en el país, analizada también en otros estudios $(9,13,14,22,27)$ sino que dan cuenta de la magnitud de su impacto diferencial por sexo, edad y entidad federativa. Estos factores sociodemográficos por sí solos, no pueden clarificar ni mucho menos determinar la causa de suicidio; sin embargo, son una importante guía para identificar la tendencia del fenómeno y conocer ciertos factores de riesgo en cuanto al perfil de la población o las características de los lugares donde se llevan a cabo los suicidios (27). Respecto a esto, se ha detectado que el principal factor de riesgo en torno al suicidio es un intento previo de suicidio (22). Sin embargo, existen otros factores asociados con esta causa de muerte, entre los que destacan: el sexo masculino; una edad menor a 40 años; personas que no están casadas, ni viven en unión libre; una menor escolaridad; el subempleo (13,31); un alto consumo de alcohol; el dolor crónico y las enfermedades, así como antecedentes familiares de suicidio; padecer algún trastorno mental principalmente depresivo (en especial la depresión mayor), de ansiedad (que combinados con los depresivos, multiplican el riesgo), de personalidad, bipolaridad, el esquizoafectivo o la esquizofrenia misma (14,32-35); y la ideación suicida, aunque en menor medida que el intento previo (15).

Adicionalmente a los factores de riesgo individuales, existen aquellos asociados con el sistema de salud y con la sociedad, entre los que destacan: dificultades para obtener acceso a la atención de salud y recibir la asistencia necesaria; la estigmatización de quienes buscan ayuda por comportamientos suicidas y la fácil disponibilidad de los medios utilizables para suicidarse (10). Aunado a ellos, por cada suicidio cometido hay muchos más intentos de suicidio, que se estima que ascienden a cifras entre 20 y 40 intentos por cada suicidio consumado (10), con una muy probable subestimación del número de intentos. Esta situación no es trivial debido a que los intentos de suicidio constituyen una gran carga social y económica, ya que cada uno tiene la posibilidad de resultar en la muerte o en la utilización de los servicios de salud para tratar las lesiones, el impacto psicológico y social del comportamiento en el individuo y su entorno, o en una lesión física o discapacidad a largo plazo, que es causa de un profundo sufrimiento psicológico (14).

Los resultados que se presentan ponen de manifiesto un panorama preocupante, no solo desde el punto de vista social, económico y de salud, sino también en cuanto a las necesidades en materia de políticas públicas (9). Resalta que el suicidio tenga escasa prioridad para los gobiernos y los tomadores de decisión dado que es una causa de muerte prevenible. Esto representa una Ilamada de atención acerca de la necesidad de implementar acciones de identificación oportuna, una estrategia integral multisectorial de prevención y el estudio detallado de los factores de riesgo asociados. La prevención del suicidio debe basarse en un conjunto de estrategias entre las que destacan: la identificación y el tratamiento de los sujetos con trastornos mentales orientado a promover la salud mental (22); incrementar el acceso a la atención de salud, promover una reducción del consumo nocivo de alcohol y de sustancias; limitar el acceso a los medios utilizables para suicidarse como el control de armas 
de fuego; promover una información responsable por parte de los medios de difusión; contar con el apoyo de la comunidad; y una mejor capacitación del personal de salud en el manejo de los trastornos mentales y el abuso de sustancias (5).

En cualquier caso, el suicidio puede considerarse no como una decisión personal, sino como un fenómeno social que obedece a ciertos factores socioculturales presentes en toda sociedad y que influyen de manera directa en la conducta suicida (27). De acuerdo con lo postulado por Durkheim, el suicidio se presenta en sociedades con desintegración, falta de cohesión y poca convivencia entre la comunidad y las instituciones por lo que se puede considerar como un reflejo de las sociedades que carecen de cohesión social, así como de sentido de pertenencia (36). Durkheim distingue cuatro categorías de suicidio: el egoísta, el anómico, el altruista y el fatalista (36). El egoísta se caracteriza por un exceso de individualismo, ante la escasa integración de las personas en la sociedad $(27,36)$. El suicidio anómico se asocia con la falta de orden social y la ausencia de normas. En cuanto al suicidio altruista, el interés del individuo está centrado en el bien de la sociedad y está dispuesto a dar su vida por el bien común. El suicidio fatalista se atribuye a la regulación excesiva de la sociedad. Estas dos últimas categorías de suicidios son poco comunes en la sociedad mexicana actual (27). Sin embargo, la disminución de lazos sociales, el creciente individualismo, la desigualdad social y la exclusión social, económica, laboral y educativa (37) del contexto mexicano actual pueden convertirse en detonantes para el desarrollo de desórdenes psiquiátricos (27), como se ha visto, uno de los principales factores de riesgo de suicidio. Es por ello que, en este contexto, los suicidios egoístas y anómicos pueden ser los más comunes en el país y los que presenten un mayor incremento en fechas recientes.

Si se acepta que los suicidios se producen como consecuencia de las problemáticas que enfrenta una población en un momento dado, la tendencia creciente de la mortalidad por esta causa observada en este estudio, así como en los realizados en el contexto mexicano $(9,13,14,22,27)$, es un parámetro de que algo grave sucede en la sociedad mexicana (10). Por lo tanto, es imperativo dedicar mayores esfuerzos a la prevención, al estudio, la detección y el tratamiento del suicidio.

Los resultados de esta investigación Ilevan a concluir que el suicidio en México se ha convertido en un problema de salud pública de gran relevancia e impacto, por lo que su estudio se debe realizar desde un enfoque multidimensional, que tome en cuenta la compleja interacción de factores biológicos, genéticos, psicológicos, sociológicos y ambientales del fenómeno $(10,33,35,38)$. Todo ello con miras a generar políticas de prevención y atención oportuna, y con base en ello buscar la disminución del impacto de esta causa de muerte en la sociedad.

\section{NOTAS FINALES}

a. El índice de marginación estatal es una medida que permite diferenciar entidades del país según el impacto global de las carencias que padece la población como resultado de la falta de acceso a diferentes oportunidades, servicios o bienes. El índice de marginación está conformado por nueve indicadores socioeconómicos y permite acercarse al conocimiento de la desigualdad regional existente de las oportunidades sociales.

b. Los otros dos supuestos del método, como lo define Arriaga (10) son: "i) Suponer que entre las dos edades elegidas para el análisis, aquellos que mueren a una edad determinada, de no haber muerto, deberían haber vivido tantos años como el promedio que vive la población que no muere a dicha edad. ii) No limitar la edad superior del análisis, y suponer que aquellos que fallecen a una edad determinada, si no hubieran muerto, habrían vivido tantos años como el resto de la población que queda viva a esa misma edad".

c. Esto implica que ${ }_{n} d_{x, j}={ }_{n} d_{x}\left({ }_{n} D_{x, j} /{ }_{n} D_{x}\right)$, donde ${ }_{n} d_{x, j}$ son las defunciones entre las edades $x$ y $x+n$ por la causa de muerte $j$ en la tabla de vida; ${ }_{n} d_{x}$ son las defunciones entre las edades $x$ y $x+n$ en la tabla de vida; $D_{x, j}$ son las defunciones registradas en las estadísticas vitales entre las edades $x$ y $x+n$ por la causa de muerte $j$; y $D_{x}$ son las defunciones registradas en las estadísticas vitales entre las edades $x$ y $x+n$. 


\section{REFERENCIAS BIBLIOGRÁFICAS}

1. Pérez S. El suicidio, comportamiento y prevención. Revista Cubana de Medicina General e Integral. 1999;15(2):196-217.

2. Nizama M. Suicidio. Revista Peruana de Epidemiología. 2011;15(2):81-85.

3. Gonzalez-Forteza C, Jiménez A. Problemática suicida: algunas consideraciones desde la investigación psicosocial: El suicidio en México. México: Pax; 2009

4. Mondragon L, Saltijeral MT, Bimbela A, Borges G. La ideación suicida y su relación con la desesperanza, el abuso de drogas y alcohol. Salud Mental. 1998;21(5):20-27.

5. Organización Mundial de la Salud. Prevención del suicidio: un imperativo global. Washington DC: OMS; 2014

6. Organización Mundial de la Salud. Informe mundial sobre la violencia y la salud. Ginebra: OMS; 2002

7. Moyano E, Barría R. Suicidio y producto interno bruto en Chile: hacia un modelo predictivo. Revista Latinoamericana de Psicología. 2006;38(2):343-359.

8. Instituto Nacional de Estadística y Geografía. Estadística de suicidios de los Estados Unidos Mexicanos 2010. México: INEGI; 2010.

9. Cárdenas R. Vidas truncadas: Mortalidad por accidentes y violencia en hombres de 15 a 29 años en México. Coyuntura Demográfica. 2014;5:23-29.

10. Arriaga E. Los años de vida perdidos: su utilización para medir el nivel y cambio de la mortalidad. Notas de Población. 1996;24(63):7-38.

11. González-Pérez G, Vega-López M, Cabrera-Pivaral C. Impacto de la violencia homicida en la esperanza de vida masculina de México. Revista Panamericana de Salud Pública. 2012;32(5):335-342.

12. Jasso R. La dimensión espacial del suicidio y su vínculo con el mercado laboral mexicano (2000-2004). Revista Facultad de Ciencias Económicas. 2013;21(1):189-216.

13. Borges G, Orozco R, Medina-Mora ME. Índice de riesgo para el intento suicida en México. Salud Pública de México. 2012;54:595-606.

14. Martínez C. Población y salud mental en México: Reflexiones y un ejercicio de aproxi- mación mediante las variaciones de la mortalidad por suicidio. Estudios Demográficos y Urbanos. 2010;25(3):663-712.

15. Hernández-Bringas $\mathrm{HH}$, Flores-Arenales $\mathrm{R}$. El suicidio en México. Papeles de Población. 2011;68:69-101.

16. Schrijvers D, Bollen J, Sabbe B. The gender paradox in suicidal behavior and its impact on the suicidal process. Journal of Affective Disorders. 2012;138:19-26

17. Saltijeral MT, Terroba G. Epidemiología del suicidio y del parasuicidio en la década de 1971 a 1980 en México. Salud Pública de México. 1987;29(4):345-360.

18. Friedmann H, Kohn R. Mortality, or probability of death, from a suicidal act in the United States. Suicide \& Life-Threatening Behavior. 2008;38:287-301.

19. Beautrais AL. Gender issues in youth suicidal behavior. Emergency Medicine. 2002;14:35-42.

20. Kanchan T, Menezes RG. Suicidal hanging in Manipal, South India - victim profile and gender differences. Journal of Forensic Legal Medicine. 2008;15:493-496.

21. Kanchan T, Menon A, Menezes RG. Methods of choice in completed suicides; gender differences and review of literature. Journal of Forensic Sciences. 2009;54(4):938-942.

22. Borges G, Orozco R, Benjet C, Medina-Mora ME. Suicidio y conductas suicidas en México: retrospectiva y situación actual. Salud Pública de México. 2010;52:292-304.

23. Puentes E, López L, Martínez T. La mortalidad por suicidios: México 1990-2001. Revista Panamericana de Salud Pública. 2004;16(2):102-109.

24. Híjar M, Rascón R, Blanco J, López M. Los suicidios en México: Características sexuales y geográficas (1979-1993). Salud Mental. 1996;19(4):14-21.

25. Canetto SS, Sakinofsky I. The gender paradox in suicide. Suicide \& Life-Threatening Behavior. 1998;28(1):1-23.

26. Möller-Leimkühler AM, Yücel M. Male depression in females? Journal of Affective Disorders. 2010;121(1-2):22-29.

27. Jiménez-Ornelas RA, Cardiel-Tellez L. El suicidio y su tendencia social en México: 1990-2011. Papeles de Población. 2013;77:205-229. 
28. Brezo J, Paris J, Turecki G. Personality traits as correlates of suicidal ideation, suicide attempts, and suicide completions: a systematic review. Acta Psychiatrica Scandinavica. 2006;113(3):180-206.

29. Strüber D, Lück M, Roth G. Sex, aggression and impulse control: an integrative account. Neurocase. 2008; 14:93-121.

30. Arias E, Blanco I. Una aproximación al entendimiento del suicidio en comunidades rurales y remotas de América Latina. Estudios Sociológicos. 2010;28(82):185-210.

31. González S, Díaz A, Ortiz S, González C, González JJ. Características psicométricas de la escala de ideación suicida de Beck en estudiantes universitarios. Salud Mental. 2000;23(6):21-30.

32. Gómez A, Lolas F, Barrera A. Los condicionantes psicosociales de la conducta suicida. Salud Mental. 1991;14(1):25-31.

33. Espinoza L, Almeida L, CortésF, Leo A, Guillermo E. Análisis de muertes por suicidio en el estado de Querétaro. Salud Mental. 2003;26(6):47-54.
34. Gutiérrez A, Contreras C. El suicidio y algunos de sus correlatos neurobiológicos: Primera parte. Salud Mental. 2008;31(4):321-329.

35. Gutiérrez A, Contreras C, Orozco R. El suicidio, conceptos actuales. Salud Mental. 2006;29(5):66-74.

36. Durkheim E. El Suicidio, estudio de sociología. Madrid: Akal; 2003.

37. Dávila-Cervantes CA, Pardo-MontañoAM.Magnitud y tendencia de la mortalidad por homicidios en Colombia y México, 2000-2011. Revista Panamericana de Salud Pública. 2014;36(1):10-16.

38. Jiménez A, González-Forteza C. Veinticinco años de investigación sobre suicidio en la Dirección de Investigaciones Epidemiológicas y Psicosociales del Instituto Nacional de Psiquiatría Ramón de la Fuente. Salud Mental. 2003;26(6):35-46.

FORMA DE CITAR

Dávila Cervantes CA, Ochoa Torres MP, Casique Rodríguez I. Análisis del impacto de la mortalidad por suicidios en México, 2000-2012. Salud Colectiva. 2015;11(4):471-484

Recibido: 19 de enero de 2015 | Versión final: 8 de junio de 2015 | Aprobado: 22 de julio de 2015

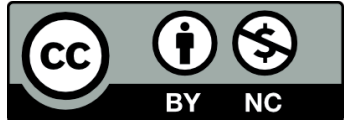

Este obra está bajo una licencia de Creative Commons Reconocimiento-NoComercial 4.0 Internacional. Reconocimiento - Permite copiar, distribuir y comunicar públicamente la obra. A cambio, se debe reconocer y citar al autor original. No Comercial - Esta obra no puede ser utilizada con finalidades comerciales, a menos que se obtenga el permiso.

http://dx.doi.org/10.18294/sc.2015.784 